\title{
Technical Report of Free Hand Pedicle Screw Placement using the Entry Points with Junction of Proximal Edge of Transverse Process and Lamina in Lumbar Spine: Analysis of 2601 Consecutive Screws
}

\author{
Chang Hyun $\mathrm{Oh}^{1}$, Seung Hwan Yoon ${ }^{2}$, Yongjung J. Kim³ \\ Dongkeun Hyun ${ }^{2}$, Hyeong-Chun Park ${ }^{2}$ \\ ${ }^{I}$ Department of Neurosurgery, Guro Teun Teun Hospital, Seoul, Korea \\ ${ }^{2}$ Department of Neurosurgery, Inha University School of Medicine, Incheon, Korea \\ ${ }^{3}$ Department of Orthopaedic Surgery, Spine Service, Columbia University College of Physicians and Surgeons, New York, NY, USA
}

Objective: A variety of different pedicle screws entry point techniques are used for the lumbar pedicle screws placement. This study reported Kim's entry point of lumbar pedicle screws with free hand technique and the accuracy of this technique. Methods: We retrospectively reviewed the 584 cases with free hand placed lumbar pedicle screw placement. The diagnosis included 491 cases with degenerative spine, 59 cases with trauma, 29 cases with metastatic disease, and 5 cases with scoliosis. A total of 2601 lumbar pedicle screws were placed, and the entry points of lumbar pedicle screws were the junction of proximal edge of transverse process and lamina. Incidence and extent of cortical breach by misplaced pedicle screw was determined by review of intra-operative and post-operative radiographs and/or computed tomography.

Results: Among the total 2601 lumbar free hand placed pedicle screws, 114 screws (4.4\%) in 79 patients (13.5\%) were repositioned screws with suspected screw malposition during operation, and 37 screws (1.4\%) in 31 patients (5.3\%) were identified as moderate to severe breaching the pedicle after post-operative imaging studies. Among the patient with malpositioned screws, 3 patients showed nerve irritation sign of the lesion, and 2 cases were symptom improved after nerve block and conservative management, and 1 case was removed the screw after the failure of the treatment.

Conclusion: Free hand pedicle screw placement based on external landmark with the junction of proximal edge of transverse process and lamina showed acceptable safety and accuracy and avoidance of radiation exposure.

Key Words: Lumbar spine $\cdot$ Pedicle screw placement $\cdot$ Free hand technique $\cdot$ Surgical technique

\section{INTRODUCTION}

Pedicle screw fixation has the advantage of utilization in the lumbar spine for superior three-column fixation without encroaching into the spinal $\mathrm{canal}^{7,12,14)}$. This advantage has been translated to superior clinical results in fracture fixation as well as in deformity correction ${ }^{13,21,29,32)}$. However, their usage has the potential for neurologic deficit, the safety margin for this techniques include the use of dorsal landmarks, lam-

- Received: Marchr 8, 2013 - Revised: March 18, 2012

- Accepted: March 19, 2012

Corresponding Author: Seung Hwan Yoon, MD

Department of Neurosurgery, Inha University Hospital, College of Medicine, Inha University, Incheon, Korea 7-206, 3-Ga, Shinheung-dong, Jung-gu, Incheon 400-711, Korea

Tel: +82-32-890-2370, Fax: +82-32-890-2374

E-mail: nsyoon@gmail.com inoforaminotomy, C-arm fluoroscopy, and computer-assisted techniques have been improved ${ }^{2-4,17,18,26,34)}$. Nevertheless, these techniques through the use of image-guided techniques require additional equipment as well as the use of fluoroscopy which increases the radiation exposure ${ }^{12}$.

Recently, the free hand technique has gained popularity. The goal of the free hand technique is to mimic as close as possible the technique of lumbar screw placement without use of any intraoperative fluoroscopy, radiography, and/or image-guided techniques ${ }^{11,14,27)}$. One of the authors reported a series in which 3400 thoraco-lumbar screws had been placed by the free hand technique without neurovascular complications, and only $6 \%$ had moderate breaches ${ }^{12,14,15)}$. Although their experience demonstrates that pedicle screws can be consistently placed in an accurate fashion using the described free hand method, it is important that each surgeon carefully review the pedicle entry point and screw direction in both sagittal and axial planes that surgeon may require to accurately place pedi- 
cle screws ${ }^{12)}$.

Generally, the pedicle entry point crosses the vertical line that forms the extension of the facet joint in line with the bony crest coming from the superior articular facet, and the horizontal line that passes through the middle of the transverse process insertion, or 1-2 $\mathrm{mm}$ below the joint line.16) The entry point of the pedicle is about $1-2 \mathrm{~mm}$ lateral from the center of the pedicle for medialization ${ }^{16}$. It may be necessary to enter the pedicle slightly more laterally, at the base of the transverse process, just as it comes up into the superior articular process to ensure the fixation is lateral enough to avoid impingement on the intact facet joint and capsule at the adjacent segment ${ }^{16}$. Many surgeons recommended various entry point and various screw placement method, and the general optimal point of screw entry is at the junction of the pars interarticularis, the midpoint of the transverse process and the inferior point of the superior articular facet as presented in Fig. $1^{16)}$.

In this study, the authors placed pedicle screws in lumbar region (L1-L5) with the use of dorsal anatomic landmarks described by one of the authors, the entry point at the junction of the proximal edge of the transverse process and lamina, where it meets the lamina and superior facet, just lateral to the midportion of the base of the superior process ${ }^{14)}$. This study aimed to evaluate the safety and accuracy of the Kim's entry point of pedicle screw placement with a free hand technique by reviewing intra-operative and post-operative imaging in the lumbar region by a single surgeon.

\section{MATERIALS AND METHODS}

From 2009 to 2011, 627 patients to our hospital with thoracolumbar regional problem were enrolled in this study. Among them, 43 patients operated by using percutaneous pedicle screws with intraoperative fluoroscopy were excluded, and 584 consecutive patients who underwent posterior stabilization utilizing 2601 transpedicular lumbar screws were analyzed (Fig. 3). The diagnosis included 491 cases with degenerative spine, 59 cases with trauma, 29 cases with metastatic disease, and 5 cases with scoliosis. The 244 patients (41.7\%) were male and the mean age was $57.5 \pm 13.9$ years (range $15-85$ years) at the time of surgery. All pedicle screws were inserted using a free hand technique with the entry points of lumbar pedicle screws at the junction of proximal edge of transverse process and lamina, similar to the technique which was reported by Kim et al. The number of screws per the lumbar level was 144 screws in L1, 258 screws in L2, 436 screws in L3, 856 screws in L4, and 907 screws in L5 (Table 1).

To objectively evaluate the position of the screws inserted into the lumbar spine, intraoperative and postoperative ante-

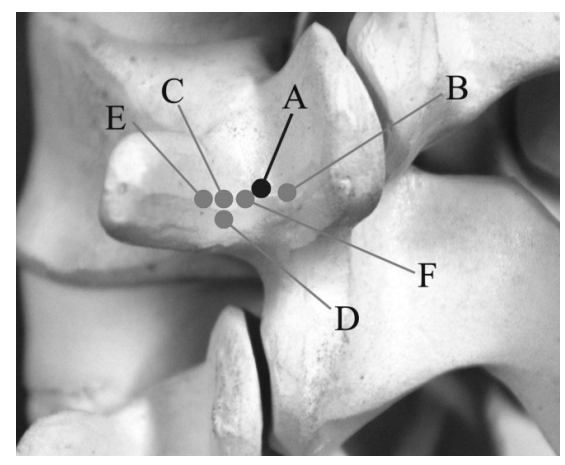

Fig. 1. The optimal point of pedicle screw entry is at the junction of the pars interarticularis, the midpoint of the transverse process and the inferior point of the superior articular facet: the point $A$ indicates the entry point of current study with the junction of the proximal edge of the transverse process and lamina; the point B indicates Roy-Camille's screw entry point; the point C indicates Magerl's method; the point D indicates Levin and Edwards method; the point $E$ indicates Weinstein's method; and the point $F$ indicates the $D$ and Zhao method.

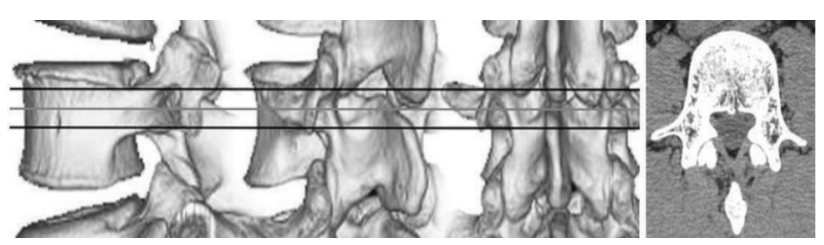

Fig. 2. Reconstructive image of the entry point of pedicle screw.

627 cases with lumbar pedicle screw placement from 2009-2012

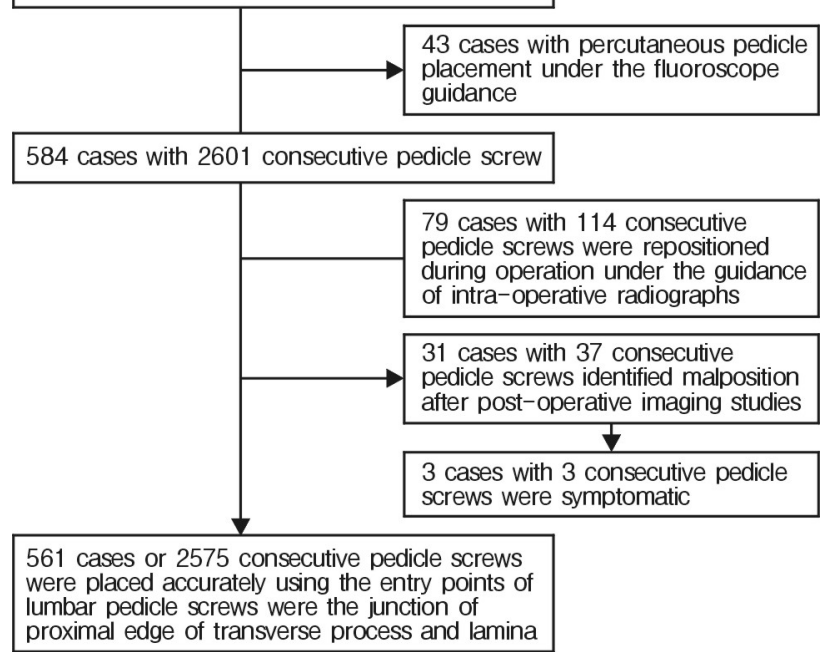

Fig. 3. Flowchart of the patients with free-hand pedicle screw placement using the Kim's entry point.

roposterior/lateral radiographs were checked, and CT scans were performed in 81 patients (365 lumbar pedicle screws) with follow-up and/or suspected screw malposition. The CT 
Table 1. The number of screws and malposition according to each anatomic lumbar level

\begin{tabular}{cccc}
\hline \hline $\begin{array}{c}\text { Spinal } \\
\text { Level }\end{array}$ & $\begin{array}{c}\text { Total No. of } \\
\text { Lumbar Pedicle } \\
\text { Screws }\end{array}$ & $\begin{array}{c}\text { Reposition of Screws } \\
\text { during Operation } \\
(\%)\end{array}$ & $\begin{array}{c}\text { Moderate to Severe } \\
\text { Breaching of Screws } \\
(\%)\end{array}$ \\
\hline L1 & 144 & $6(4.2)$ & $3(2.1)$ \\
L2 & 258 & $6(2.3)$ & $4(1.6)$ \\
L3 & 436 & $25(5.7)$ & $7(1.6)$ \\
L4 & 856 & $42(4.9)$ & $10(1.2)$ \\
L5 & 907 & $35(3.9)$ & $13(1.4)$ \\
Total & 2601 & $114(4.4)$ & $37(1.4)$ \\
\hline
\end{tabular}

scans (axial and sagittal) were examined by a surgeon and a radiologist to evaluate the position of screws according to the classification of Learch and Wiesner ${ }^{19,35)}$. It is worthy to mention that the ideal position of the screw is achieved when the screw lies in the middle of the pedicle in both axial and sagittal reconstruction CT scans. In this classification, there are four main categories for screw misplacement: encroachment, the pedicle cortex could not be visualised; minor penetration, the screw trajectory was $<3 \mathrm{~mm}$ outside the pedicular boundaries; moderate penetration, the screw trajectory was 3-6 mm outside the pedicular boundaries; and severe penetration, the screw trajectory was $>6 \mathrm{~mm}$ outside the pedicular boundaries. When there was disagreement on position of the screws in the pedicle, it was counted according to at least two concordant reads. The authors compared the sensitivity and specificity of intraoperative radiograph confirmation to detect the malposition of screws.

The surgical technique of "free hand" pedicle screw placement can be broken down into specific steps that are repeated at each level, and is very similar to the technique reported by Kim et al. ${ }^{14)}$ The first important component of successful pedicle screw placement is meticulous exposure of the posterior elements. The spine is exposed to the tips of the transverse processes bilaterally, staying strictly subperiosteal to reduce bleeding. The facet joints must be thoroughly cleaned of soft tissue. With an osteotome, remove the inferior 3-5 $\mathrm{mm}$ of the inferior facet and scrape the cartilage on top of the superior facet to enhance the intra-articular arthrodesis. In patients with moderate to severe hypertrophied facet joint, the facet joint should be cleaned to establish the entry point. The entry point of the lumbar pedicle screw is at the junction of the proximal edge of the transverse process and lamina, where it meets the lamina and superior facet, just lateral to the midportion of the base of the superior process (Fig. 1, 2).

An acorn tipped burr is utilized to create posterior cortical breach, approximately $5 \mathrm{~mm}$ in depth. A pedicle "blush" may be visualized suggesting entrance into the cancellous bone at the base of the pedicle. The Lenke's probe ( $2 \mathrm{~mm}$ blunt-tipped, slightly-curved pedicle finder) is placed in the base of the pedi- cle searching for a cancellous "soft spot" indicating entrance to the pedicle. The probe is pointed lateral as a safety measure to avoid medial wall perforation. After inserting the tip is pointed medial to face the vertebral body with an ultimate depth averaging $30-40 \mathrm{~mm}$ for the lumbar region. Rotate the finder $180-360^{\circ}$ to make room for the screw after advancing the finder to the approximate length of the desired screw. Make sure you feel bone the entire length of the pedicle and body. Probing of the pedicle should proceed in a smooth and consistent manner with a snug feel. Any sudden advancement of the probe suggests penetration into soft tissue and thus a pedicle wall or vertebral body violation. These should be investigated immediately in order to possibly salvage the pedicle and avoid complications. The surgeon must use caution as the anterior and lateral vertebral body cortices are not very strong and may be easily penetrated by the prove tip.

Once the pedicle seeker is removed, the tract is visualized to make sure that only blood is coming out and not cerebrospinal fluid. Next, a flexible ball-tipped pedicle sounding or palpating device is utilized to palpate five distinct bony borders: a floor and four walls (medial, lateral, superior, and inferior). Pay special

attention to the junction of the middle and upper portions of the tract, as this is the region of the pedicle where the spinal canal is located and the pedicle isthmus is located. This is an absolutely critical step, whereby inadvertent deep (anterior), medial, lateral, or more rare superior and inferior pedicle breaches can be identified. At this point, if a soft tissue breach is palpated, there may be an opportunity to redirect the screw into an appropriate position into the pedicle so that complete intraosseous borders can be obtained. If any wall including the medial has been breached, the pedicle may be salvageable. Otherwise, place bone wax in the pedicle hole to eliminate the bleeding and reangle the pedicle finder with a more appropriate trajectory. With the sounder in the base of the anticipated pedicle tract after confirming five intraosseous borders, mark the length of the tract with a hemostat and measure it.

The pedicle tract is undertapped with a $0.5 \mathrm{~mm}$ less diameter tap than the intended screw. Following this, the pedicle tract is palpated again to make sure that the five osseous borders are intact. This second palpation will often allow palpation of distinct bony ridges confirming intraosseous position, and the tract length is remeasured with a hemostat. Comparethis measurement directly adjacent to the screw to be placed to ensure appropriate screw length. Place the screw slowly down the pedicle into the body in the same alignment to confirm it is threaded properly and allow for viscoelastic expansion.

It is imperative that the surgeon document intraosseous placement via fluoroscopy and/or radiography. The coronal 

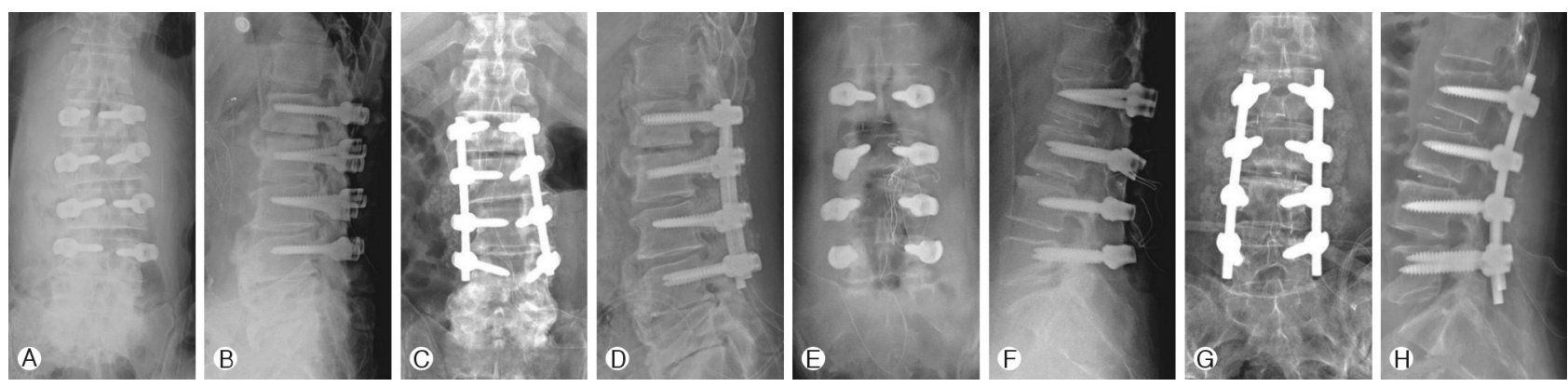

Fig. 4. Confirmation of intraosseous screw placement using intraoperative anteroposterior/lateral radiographs: $A, B, E$ and $F$, intraoperative radiographs including all inserted screws are performed after all screws are placed; C, D, $G$ and $H$, postoperative radiographs which was checked within 7 days after surgery

plane radiograph is evaluated for the harmonious position of all screws (Fig. 4). In cases of shorter or longer screws compared to adjacent screws, the screw length as viewed on the lateral radiograph is mandatory to check for harmonious position. On the lateral radiograph, the screws should be parallel to the superior endplates and not extending past the anterior border of the vertebral body. If the malposition of pedicle screws were suspected in intraoperative images, the surgeon should remove the suspected screw, and repalpate five distinct bony borders by using a flexible ball-tipped pedicle sounding or palpating device. The authors reinserted the pedicle screw when the bony border was intact, or reproved with corrected trajectory when the border was not intact.

\section{RESULTS}

A total of 584 patients received free-hand pedicle screw placement in the lumbar spine at our institution over the 3year period reviewed. As shown in Fig. 3, total 2601 screws were inserted with the entry points of lumbar pedicle screws were the junction of proximal edge of transverse process and lamina, 114 screws (4.4\%) in 79 patients (13.5\%) were repositioned screws with suspected screw malposition during operation, and 37 screws (1.4\%) in 31 patients (5.3\%) were identified as moderate to severe breaching the pedicle after post-operative imaging studies. The repositioned or malpositioned screws according to the lumbar level were shown in Table 2. The repositioned screws during operation were 6 screws (4.2 \%) in L1, 6 screws (2.3\%) in L2, 25 screws (5.7\%) in L3, 42 screws (4.9\%) in L4, and 35 screws (3.9\%) in L4 level. The confirmed malpositioned screws were 3 screws $(2.1 \%)$ in L1, 4 screws (1.6\%) in L2, 7 screws (1.6\%) in L3, 10 screws $(1.2$ $\%)$ in L4, and 13 screws (1.4\%) in L5 level. Among the patient with malpositioned screws, 3 patients showed nerve irritation sign of the lesion, and 2 cases were symptom improved after
Table 2. The breaching screws among 81 patients (365 screws) with postoperative radiographs and computed tomography (88.4 $\%$ of sensitivity and $86.5 \%$ of specificity of postoperative radiographs)

\begin{tabular}{|c|c|c|c|}
\hline & $\begin{array}{c}\text { Good } \\
\text { positioned } \\
\text { screws in CT }\end{array}$ & $\begin{array}{l}\text { Moderate to } \\
\text { severe breaches } \\
\text { in CT }\end{array}$ & s Total \\
\hline $\begin{array}{l}\text { Suspected good position } \\
\text { in radiographs }\end{array}$ & 290 & 5 & 295 \\
\hline $\begin{array}{l}\text { Suspected malposition in } \\
\text { radiographs }\end{array}$ & 38 & 32 & 70 \\
\hline Total & 328 & 37 & 365 \\
\hline
\end{tabular}

nerve block and conservative management, and 1 case was removed the screw after the failure of the treatment.

Among the total 584 patients, CT scans were performed in 81 patients (13.9\%) and 365 lumbar pedicle screws (14.0\%) with follow-up and/or suspected screw malposition. The accuracy of checking the pedicle screw placement by radiographs were showed as $88.4 \%$ of sensitivity and $86.5 \%$ of specificity compared to the CT scans.

\section{DISCUSSION}

The technique for pedicle screw instrumentation of the spine has gone through significant progress over the last two decades $^{23,28)}$. The pedicle screws placement was initially used in the lumbar spine ${ }^{21)}$. Besides offering an overall construct rigidity, pedicular screws have several advantages over traditional hook and rod constructs: they allow the stability necessary for spinal arthrodesis and improve deformity correction due to its three-column control over the spinal elements ${ }^{23}$. Pedicle screw instrumentation also obviates the need to place instrumentation within the spinal canal thus lessens the risk of neural injury ${ }^{4,26,34)}$. Moreover, the placement of pedicle screws is independent of facet or laminar integrity and, thus, 
has been extremely useful to a varied range of spinal pathologies, such as traumatic, degenerative, oncologic, as well as deformity correction ${ }^{2,10,20,22,24)}$.

The free-hand technique for placement of pedicle instrumentation relies completely on the use of visible as well as palpable anatomic landmarks for accurate pedicle screw placement ${ }^{23)}$. It is dependent on a clear exposition and identification of the posterior bony landmarks, including the lateral border of the pars interarticularis, the entire transverse process and the caudal and cephalad facet joints ${ }^{16}$. In the last few years several anatomic studies have been performed to detail the complex morphometry and three-dimensional anatomy of the lumbar pedicles, ${ }^{6,8}$. In the lumbar spine an osteotomy (partial facetectomy of the infero-lateral one-third of the inferior articular process of the superior vertebrae) is usually performed for three reasons: It enables the identification of the exact limit between the superior and inferior articular processes; it facilitates the correct identification of the ideal initial perforation site; it decreases the amount of cortical bone (which may make the initial perforation difficult, especially in the presence of hypertrophic facets); and finally it provides a smooth bony surface for final allocation of the head of the screw $^{23)}$. The general entry point is in the intersection between a line that passes just laterally to the inferior articular process and a line which bisects the transverse process ${ }^{27}$. At S1, the entry point is at the infero-lateral margin of the basis of the superior articular process of the sacrum ${ }^{23}$. The direction of the screws follows the axis of the pedicle. It is slightly oblique towards the midline (on an average $10-15^{\circ}$ ). Its angular value is variable according to the individual. The obliquity towards the sagital plane can be preoperatively checked by various imaging studies such as CT scan or MRI. The length of the screws in the lumbosacral region varies from $40-45 \mathrm{~mm}$ for $\mathrm{L} 1$ to L4, $45-50 \mathrm{~mm}$ for $\mathrm{L} 5$, and $35-40 \mathrm{~mm}$ for S1. After the initial perforation, the border of pedicle palpation by ball tip and gearshift probe, as well as tapping, are utilized in order to verify the presence of possible pedicle violations as we described above. After the placement of pedicle screws the final construct is connected such as rods, 3D heads, and loction caps.

The overall reported rates of correct pedicle screw placement is extremely variable in the surgical literature, ${ }^{5,9,33)}$. Although there are anecdotal articles reporting "miss" rates as high as $55 \%$ in cadaveric series of free-hand technique placement of pedicle screw in the thoracic spine ${ }^{36}$, the accuracy of freehand technique of lumbar spine pedicle screw placement is excellent by the unique anatomic characteristics such as more thicker of pedicle size than thoracic spine ${ }^{14)}$. The reported the accuracy of lumbar pedicle screw placement were summarized in Table 3. Overall accuracy of lumbar pedicle screw placement were $93.5-99.1 \%$ with different entry point of pedicle screws. Su et al. ${ }^{31)}$ used Margel's technique (the entry point of lumbar spine lies at the junction of the lateral border of the superior articular process and the bisector of the transverse process), and reported $93.5 \%$ of accuracy in lumbar pedicle screws among scoliosis cases. The Roy-Camille method (the starting point for screw insertion was determined to be the intersection between the midlines of the facet joint and transverse process) was used by Silbermann et al. ${ }^{30)}$ and the accuracy was reported as $94.1 \%$. The entry point of the junction of the pars interarticularis with the transverse process and the mamillary process/superior articular process of the segment of interest (similar to Du and Chao method) was selected by Beck et al. ${ }^{1)}$ and Parkter et al. ${ }^{2)}$ and reported $96.8 \%$ and

Table 3. The reported the accuracy of lumbar pedicle screw placement

\begin{tabular}{|c|c|c|c|c|c|}
\hline Authors (Publish year) & Technique & Cases & $\begin{array}{l}\text { Total } \\
\text { screws }\end{array}$ & $\begin{array}{l}\text { Lumbar } \\
\text { screws }\end{array}$ & $\begin{array}{l}\text { Lumbar Pedicle } \\
\text { Screw Accuracy (\%) }\end{array}$ \\
\hline Su et al $(2012)^{33}$ & Margel's technique $^{a}$ & Scoliosis & 146 & 46 & 93.5 \\
\hline Silbermann et al $(2011)^{34}$ & Roy-Camille $e^{b}$ & Various spine disease & 152 & 130 & 94.1 \\
\hline Beck et al $(2009)^{35}$ & $\begin{array}{l}\text { Similar Du and Chao method } \\
\text { cwithintraoperativeimagingconfirm }\end{array}$ & $\begin{array}{l}\text { fracture of thoracolumbar } \\
\text { spine }\end{array}$ & 414 & 220 & 96.8 \\
\hline Karapinar et al $(2008)^{4}$ & Levin and Edwards method ${ }^{d}$ & Various spine disease & 640 & 343 & 97.7 \\
\hline Parker et al $(2011)^{36}$ & Similar Du and Chao method ${ }^{\mathrm{e}}$ & Various spine disease & 6,816 & 3,373 & 99.1 \\
\hline Present study & Kim's method ${ }^{f}$ & Various spine disease & 2,601 & 2,601 & 98.6 \\
\hline
\end{tabular}

a) The entry point of lumbar spine lies at the junction of the lateral border of the superior articular process and the bisector of the transverse process; b) the starting point for screw insertion was determined to be the intersection between the midlines of the facet joint and transverse process; c) Lumbar starting point was the junction of mammillary process, inferior aspect of transverse process and pars interarticularis; d) The starting point for pedicle screw was the intersection of the transverse process with the junction of the middle and lateral one third of the corresponding superior articular process; e) junction of the pars interarticularis with the transverse process and the mamillary process/superior articular process of the segment of interest; $f$ the entry point at the junction of the proximal edge of the transverse process and lamina, where it meets the lamina and superior facet, just lateral to the midportion of the base of the superior process 
99.1\% of lumbar pedicle screw accuracy. The Levin and Edward method (the starting point for pedicle screw was the intersection of the transverse process with the junction of the middle and lateral one third of the corresponding superior articular process) was reported as $97.7 \%$ of accuracy ${ }^{13}$.

Traditional lumbar entry point techniques used the transverse process and facet joint as important anatomical land marks (Fig. 1). The Margel's method used the entry point indicted by the intersection of lateral border of facet joint (superior articular process) and bisect line of the transverse process. The Roy-Camille's entry point was also used the prolongated line of the facet joint and middle of the transverse process line. So, the clinical entry point with hypertrophied facet joint could not easily accessible in traditional entry point (Fig. 2). But, the new entry point which was demonstrated in this study used the entry point as the junction of proximal edge of transverse process and lamina. This landmarks were not changed by hypertrophied facet joint, and easily accessible by drilling of superior articular process.

In this study, there were some limitations. First of all, all cases and screws were not reviewed the postoperative CT scans. The Postoperative CT checked in only 81 cases (365 lumbar pedicle screws) for follow-up and/or suspected screw malposition. But, the postoperative radiographs were taken to all cases, and it showed good sensitivity (88.4\%) and specificity (86.5\%). So, the real number of malposition screws could different to current results, but not significantly different. Some well designed study was warranted to solve this problem.

\section{CONCLUSION}

Free hand pedicle screw placement based on external landmark with the junction of proximal edge of transverse process and lamina showed acceptable safety and accuracy and avoidance of radiation exposure.

\section{REFERENCES}

1. Beck M, Mittlmeier T, Gierer P, Harms C, Gradl G: Benefit and accuracy of intraoperative 3D-imaging after pedicle screw placement: a prospective study in stabilizing thoracolumbar fra- ctures. Eur Spine J 18:1469-1477, 2009

2. Boachie-Adjei O, Girardi FP, Bansal M, Rawlins BA: Safety and efficacy of pedicle screw placement for adult spinal deformity with a pedicle-probing conventional anatomic technique. J Spinal Disord 13:496-500, 2000

3. Bransford R, Bellabarba C, Thompson JH, Henley MB, Mirza SK, Chapman JR: The safety of fluoroscopically-assisted thoracic pedicle screw instrumentation for spine trauma. J Trauma 60: 1047-1052, 2006
4. Carl AL, Khanuja HS, Gatto CA, Matsumoto M, vomLehn J, Schenck J, et al: In vivo pedicle screw placement: image-guided virtual vision. J Spinal Disord 13:225-229, 2000

5. Castro WH, Halm H, Jerosch J, Malms J, Steinbeck J, Blasius S: Accuracy of pedicle screw placement in lumbar vertebrae. Spine (Phila Pa 1976) 21:1320-1324, 1996

6. Cinotti G, Gumina S, Ripani M, Postacchini F: Pedicle instrumentation in the thoracic spine. a morphometric and cadaveric study for placement of screws. Spine (Phila Pa 1976) 24:114-119, 1999

7. Cotrel Y, Dubousset J, Guillaumat M: New universal instrumentation in spinal surgery. Clin Orthop Relat Res 227:10-23, 1988

8. Ebraheim NA, Jabaly G, Xu R, Yeasting RA: Anatomic relations of the thoracic pedicle to the adjacent neural structures. Spine (Phila Pa 1976) 22:1553-1556, 1997

9. Farber GL, Place HM, Mazur RA, Jones DE, Damiano TR: Accuracy of pedicle screw placement in lumbar fusions by plain radiographs and computed tomography. Spine (Phila Pa 1976) 20: 1494-1499, 1995

10. Fernández-Fairen M, Sala P, Ramírez H, Gil J: A prospective randomized study of unilateral versus bilateral instrumented posterolateral lumbar fusion in degenerative spondylolisthesis. Spine (Phila Pa 1976) 32:395-401, 2007

11. Hamill CL, Lenke LG, Bridwell KH, Chapman MP, Blanke K, Baldus C: The use of pedicle screw fixation to improve correction in the lumbar spine of patients with idiopathic scoliosis. Is it warranted? Spine (Phila Pa 1976) 21:1241-1249, 1996

12. Hyun SJ, Kim YJ, Cheh G, Yoon SH, Rhim SC: Free hand pedicle screw placement in the thoracic spine without any radiographic guidance: technical note, a cadaveric study. J Korean Neurosurg Soc 51:66-70, 2012

13. Karapinar L, Erel N, Ozturk H, Altay T, Kaya A: Pedicle screw placement with a free hand technique in thoracolumbar spine: is it safe? J Spinal Disord Tech 21:63-67, 2008

14. Kim YJ, Lenke LG, Bridwell KH, Cho YS, Riew KD: Free hand pedicle screw placement in the thoracic spine: is it safe? Spine (Phila Pa 1976) 29:333-342, 2004

15. Kim YJ, Lenke LG, Cheh G, Riew KD: Evaluation of pedicle screw placement in the deformed spine using intraoperative plain radiographs: a comparison with computerized tomography. Spine (Phila Pa 1976) 30:2084-2088, 2005

16. Korean Spinal Neurosurgery Society: Surgical atlas of spine, ed 1. Seoul: Koon Ja pp267-276, 2010

17. Kuntz $\mathrm{C} 4^{\text {th }}$, Maher PC, Levine NB, Kurokawa R: Prospective evaluation of thoracic pedicle screw placement using fluoroscopic imaging. J Spinal Disord Tech 17:206-214, 2004

18. Laine T, Schlenzka D, Mäkitalo K, Tallroth K, Nolte LP, Visarius $\mathrm{H}$ : Improved accuracy of pedicle screw insertion with computer-assisted surgery. a prospective clinical trial of 30 patients. Spine (Phila Pa 1976) 22:1254-1258, 1997

19. Learch TJ, Massie JB, Pathria MN, Ahlgren BA, Garfin SR: Assessment of pedicle screw placement utilizing conventional radiography and computed tomography: a proposed systematic approach to improve accuracy of interpretation. Spine (Phila Pa 1976) 29:767-773, 2004

20. Liljenqvist UR, Halm HF, Link TM: Pedicle screw instrumentation of the thoracic spine in idiopathic scoliosis. Spine (Phila $\mathrm{Pa}$ 1976) 22:2239-2245, 1997 
21. Liljenqvist UR, Link TM, Halm HF: Morphometric analysis of thoracic and lumbar vertebrae in idiopathic scoliosis. Spine (Phila Pa 1976) 25:1247-1253, 2000

22. LüFX, Huang Y, Zhang Q, Shi FL, Zhao DS, Hu Q: Pedicle screw fixation against burst fracture of thoracolumbar vertebrae. Chin J Traumatol 10:349-352, 2007

23. Mattei TA, Meneses MS, Milano JB, Ramina R: "Free-hand" technique for thoracolumbar pedicle screw instrumentation: critical appraisal of current "state-of-art". Neurol India 57:715721, 2009

24. Mazel C, Hoffmann E, Antonietti P, Grunenwald D, Henry M, Williams J: Posterior cervicothoracic instrumentation in spine tumors. Spine (Phila Pa 1976) 29:1246-1253, 2004

25. Parker SL, McGirt MJ, Farber SH, Amin AG, Rick AM, Suk I, et al: Accuracy of free-hand pedicle screws in the thoracic and lumbar spine: analysis of 6816 consecutive screws. Neurosurgery 68:170-178, 2011

26. Rampersaud YR, Pik JH, Salonen D, Farooq S: Clinical accuracy of fluoroscopic computer-assisted pedicle screw fixation: a CT analysis. Spine (Phila Pa 1976) 30:E183-190, 2005

27. Reidy DP, Houlden D, Nolan PC, Kim M, Finkelstein JA: Evaluation of electromyographic monitoring during insertion of thoracic pedicle screws. J Bone Joint Surg Br 83:1009-1014, 2001

28. Roy-Camille R, Sailant G, Mazel C: Plating of thoracic, thoracolumbar, and lumbar injuries with pedicle screw plates. Orthop Clin North Am 17:147-159, 1986

29. Sarlak AY, Tosun B, Atmaca H, Sarisoy HT, BuluçL: Evaluation of thoracic pedicle screw placement in adolescent idiopathic scoli- osis. Eur Spine J 18:1892-1897, 2009

30. Silbermann J, Riese F, Allam Y, Reichert T, Koeppert H, Gutberlet $\mathrm{M}$ : Computer tomography assessment of pedicle screw placement in lumbar and sacral spine: comparison between free-hand and o-arm based navigation techniques. Eur Spine J 20:875881, 2011

31. Su P, Zhang W, Peng Y, Liang A, Du K, Huang D: Use of computed tomographic reconstruction to establish the ideal entry point for pedicle screws in idiopathic scoliosis. Eur Spine J 21: 23-30, 2012

32. Suk SI, Kim WJ, Lee SM, Kim JH, Chung ER: Thoracic pedicle screw fixation in spinal deformities: are they really safe ? Spine (Phila Pa 1976) 26:2049-2057, 2001

33. Vaccaro AR, Rizzolo SJ, Balderston RA, Allardyce TJ, Garfin SR, Dolinskas C, et al: Placement of pedicle screws in the thoracic spine. part II: an anatomical and radiographic assessment. J Bone Joint Surg Am 77:1200-1206, 1995

34. Weinstein JN, Spratt KF, Spengler D, Brick C, Reid S: Spinal pedicle fixation: reliability and validity of roentgenogram-based assessment and surgical factors on successful screw placement. Spine (Phila Pa 1976) 13:1012-1018, 1988

35. Wiesner L, Kothe R, Ruther W: Anatomic evaluation of two different techniques for the percutaneous insertion of pedicle screws in the lumbar spine. Spine (Phila Pa 1976) 24:1599-1603, 1999

36. Xu R, Ebraheim NA, Ou Y, Yeasting RA: Anatomic considerations of pedicle screw placement in the thoracic spine: roycamille technique versus open-lamina technique. Spine (Phila Pa 1976) 23:1065-1068, 1998 